\title{
Skin diseases among elderly inhabitants of Bialystok, Poland
}

This article was published in the following Dove Press journal:

Clinical Interventions in Aging

8 December 2015

Number of times this article has been viewed

\section{Mateusz Cybulski \\ Elzbieta Krajewska-Kulak}

Department of Integrated Medical Care, Faculty of Health Sciences, Medical University of Bialystok, Bialystok, Poland
Correspondence: Mateusz Cybulski Department of Integrated Medical Care, Faculty of Health Sciences, Medical University of Bialystok, 7A Sklodowskiej-Curie Street, Bialystok 15-096, Poland

$\mathrm{Tel}+48856865103$

Fax +48 857485528

Email mateusz.cybulski@umb.edu.pl
Purpose: The aim of the study was to assess the most frequent skin diseases in people over 60 years old among residents of a public nursing home and students of the University of the Third Age in Bialystok.

Subjects and methods: The study was carried out from April to June 2015 in Bialystok, in two groups: 100 residents of a public nursing home and 100 participants of the University of the Third Age, aged over 60 years, using a method of diagnostic survey with the authors' anonymous questionnaire.

Results: A total of $30.5 \%$ of respondents $(n=61)$ had been treated due to skin diseases, most frequently for 6-10 years (26.2\%). Fungal infection, psoriasis, and atopic dermatitis were the most frequent dermatological diseases among the study elderly. The sites affected most frequently with these diseases were upper and lower extremities and the face. A majority of the examined $(63.9 \%)$ visited a dermatologist, but only when it was necessary.

Conclusion: Skin diseases constitute a significant health problem among seniors. The elderly should be educated about healthy lifestyle, preventing the development of fungal infections. It is necessary to encourage seniors to visit dermatologists, seeking professional advice.

Keywords: skin diseases, elderly, fungal infections, psoriasis

\section{Introduction}

The aging process is determined by numerous intrinsic and extrinsic factors and affects all body parts and tissues, including the skin. In the literature, ${ }^{1,2}$ the skin-aging process determines the development of numerous skin diseases. It is estimated that every 14 th visit at the doctor's surgery is associated with skin diseases. ${ }^{3}$

Excessive predisposition to dryness is one of typical consequences of aging, which may lead to excessive dryness of the skin, affected additionally by environmental factors, resulting in intensification of itching among elderly patients. ${ }^{4}$ It is underlined ${ }^{5,6}$ that skin lesions may reflect disease processes occurring in the systems and body parts and have a correlation with therapeutic preparations, which in consequence may generate more frequent skin problems in the elderly, determined additionally by many coexisting diseases and medications taken simultaneously.

Skin diseases, typical of the senior age, are usually associated with impaired vascularity and skin symptoms of autoimmune processes, viral infections, and inflammations in the organism. ${ }^{3,6,7}$ Various types of eczema, vesicular diseases, stasis dermatitis, bedsores, rosacea, lichen sclerosus, and herpes zoster are statistically most frequently found in seniors. ${ }^{3,6,7}$

The aim of this study was to assess the most frequent skin diseases in people over 60 years old among residents of a public nursing home (PNH) and students of the University of the Third Age (U3A) in Bialystok, Poland. 


\section{Subjects and methods Participants}

The research was conducted in two groups among 100 residents of a PNH at 9 Swierkowa Street in Bialystok and among 100 students of the U3A in Bialystok. The only criterion for the selection group for the study was age over 60 years old. A total of 200 people over 60 years old were included in the study.

\section{Measurements and procedure}

This was a cross-sectional study. A method of diagnostic survey using the authors' anonymous survey questionnaire was applied in the study. It consisted of 35 questions, both open and closed, one or multiple choice, and a review of patients' dermatological histories. The questions concerned sociodemographic characteristics (sex, marital status, education, financial status), the incidence of skin diseases among the examined (treatment due to skin diseases confirmed by dermatological examinations, length of treatment, the name of the skin disease, the site of lesions, frequency of visits at outpatient dermatological departments, family history of dermatological diseases).

\section{Procedure and ethical considerations}

The study was performed from April to June 2015. The research conformed with the Good Clinical Practice guidelines, and the procedures followed were in accordance with the Helsinki Declaration of 1975, as revised in 2000 (concerning the ethical principles for the medical community and forbidding release of the name of the patient, initials, or the hospital evidence number), and with the ethical standards of the institutional committee on human experimentation (statute from the Bioethics Committee of the Medical University in Bialystok R-I-002/417/2014). All the patients received an oral invitation to participate in this research and signed an informed consent form.

\section{Statistical analysis}

All data obtained during the study were compiled using Microsoft Excel 2010. Statistical analysis was completed by applying the $\chi^{2}$-test. Statistical hypotheses were verified at the $P=0.05$ significance level. Calculations were completed using the Statistica Data Miner + QC PL program.

\section{Results}

The baseline characteristics of the study population are shown in Table 1. Skin disease confirmed by a dermatologist was reported in $30.5 \%$ of respondents $(n=61)$, including $34.0 \%$ of $\mathrm{PNH}$ residents and $27.0 \%$ of $\mathrm{U} 3 \mathrm{~A}$ students. Therefore, 61
Table I Baseline characteristics of the study population

\begin{tabular}{|c|c|c|c|}
\hline Variables & PNH residents & U3A students & Total \\
\hline \multicolumn{4}{|l|}{ Age } \\
\hline \multicolumn{4}{|l|}{$6 I-70$ years } \\
\hline $\mathrm{n}$ & 43 & 39 & 82 \\
\hline$\%$ & $43 \%$ & $39 \%$ & $41 \%$ \\
\hline \multicolumn{4}{|l|}{$>70$ years } \\
\hline $\mathrm{n}$ & 57 & 61 & 118 \\
\hline$\%$ & $57 \%$ & $61 \%$ & $59 \%$ \\
\hline \multicolumn{4}{|c|}{ Marital status } \\
\hline \multicolumn{4}{|l|}{ Married } \\
\hline $\mathrm{n}$ & 10 & 50 & 60 \\
\hline$\%$ & $10 \%$ & $50 \%$ & $30 \%$ \\
\hline \multicolumn{4}{|c|}{ Widow/widower } \\
\hline $\mathrm{n}$ & 47 & 38 & 85 \\
\hline$\%$ & $47 \%$ & $38 \%$ & $42.5 \%$ \\
\hline \multicolumn{4}{|l|}{ Single } \\
\hline $\mathrm{n}$ & 26 & 4 & 30 \\
\hline$\%$ & $26 \%$ & $4 \%$ & $15 \%$ \\
\hline \multicolumn{4}{|l|}{ Divorced } \\
\hline $\mathrm{n}$ & 17 & 7 & 24 \\
\hline$\%$ & $17 \%$ & $7 \%$ & $12 \%$ \\
\hline \multicolumn{4}{|l|}{ Separated } \\
\hline $\mathrm{n}$ & 0 & 1 & I \\
\hline$\%$ & 0 & $1 \%$ & $0.5 \%$ \\
\hline \multicolumn{4}{|c|}{ Financial status } \\
\hline \multicolumn{4}{|c|}{ Very good } \\
\hline $\mathrm{n}$ & 4 & 0 & 4 \\
\hline$\%$ & $4 \%$ & 0 & $2 \%$ \\
\hline \multicolumn{4}{|l|}{ Good } \\
\hline $\mathrm{n}$ & 21 & 58 & 79 \\
\hline$\%$ & $21 \%$ & $58 \%$ & $39.5 \%$ \\
\hline \multicolumn{4}{|l|}{ Average } \\
\hline $\mathrm{n}$ & 54 & 36 & 90 \\
\hline$\%$ & $54 \%$ & $36 \%$ & $45 \%$ \\
\hline \multicolumn{4}{|l|}{ Rather bad } \\
\hline $\mathrm{n}$ & 12 & 0 & 12 \\
\hline$\%$ & $12 \%$ & 0 & $6 \%$ \\
\hline \multicolumn{4}{|l|}{ Bad } \\
\hline$n$ & 9 & 0 & 9 \\
\hline$\%$ & $9 \%$ & 0 & $4.5 \%$ \\
\hline \multicolumn{4}{|l|}{ Hard to say } \\
\hline $\mathrm{n}$ & 4 & 2 & 6 \\
\hline$\%$ & $4 \%$ & $2 \%$ & $3 \%$ \\
\hline Level of ec & & & \\
\hline Higher & & & \\
\hline $\mathrm{n}$ & 15 & 50 & 65 \\
\hline$\%$ & $15 \%$ & $50 \%$ & $32.5 \%$ \\
\hline Secondary & & & \\
\hline $\mathrm{n}$ & 26 & 44 & 70 \\
\hline$\%$ & $26 \%$ & $44 \%$ & $35 \%$ \\
\hline Technical & & & \\
\hline $\mathrm{n}$ & 12 & 5 & 17 \\
\hline$\%$ & $12 \%$ & $5 \%$ & $8.5 \%$ \\
\hline Vocational & & & \\
\hline $\mathrm{n}$ & 27 & 1 & 28 \\
\hline$\%$ & $27 \%$ & $1 \%$ & $14 \%$ \\
\hline Primary & & & \\
\hline $\mathrm{n}$ & 20 & 0 & 20 \\
\hline$\%$ & $20 \%$ & 0 & $10 \%$ \\
\hline Total & & & \\
\hline$n$ & 100 & 100 & 200 \\
\hline$\%$ & $100 \%$ & $100 \%$ & $100 \%$ \\
\hline
\end{tabular}

Abbreviations: $\mathrm{PNH}$, public nursing home; U3A, University of the Third Age. 
Table 2 Morbidity of skin disease in the study population

\begin{tabular}{|c|c|c|c|c|}
\hline $\begin{array}{l}\text { Morbidity of } \\
\text { skin disease }\end{array}$ & $\begin{array}{l}\text { PNH } \\
\text { residents }\end{array}$ & $\begin{array}{l}\text { U3A } \\
\text { students }\end{array}$ & Total & $P$-value \\
\hline \multicolumn{4}{|c|}{ Are you being treated due to any skin disease? } & 0.282 \\
\hline \multicolumn{5}{|l|}{ Yes } \\
\hline$n$ & 34 & 27 & 61 & \\
\hline$\%$ & $34 \%$ & $27 \%$ & $30.5 \%$ & \\
\hline \multicolumn{5}{|l|}{ No } \\
\hline $\mathrm{n}$ & 66 & 73 & 139 & \\
\hline$\%$ & $66 \%$ & $73 \%$ & $69.5 \%$ & \\
\hline \multicolumn{5}{|l|}{ Total } \\
\hline $\mathrm{n}$ & 100 & 100 & 200 & \\
\hline$\%$ & $100 \%$ & $100 \%$ & $100 \%$ & \\
\hline
\end{tabular}

Abbreviations: $\mathrm{PNH}$, public nursing home; U3A, University of the Third Age.

of 200 people were included in the next stage of the study. Table 2 shows detailed results.

More than a quarter of respondents (26.2\%), including ten PNH residents (29.4\%), had been treated due to skin diseases for $6-10$ years. A total of $23 \%$ of the questioned had been treated for 1-5 years (the most frequent length of treatment among U3A participants [29.6\%]). The same percentage (23\%) was found for all those examined for treatment of 11-15 years. A total of $16.4 \%$ of the questioned had been treated for longer than 15 years, and the remaining $11.5 \%$ less than a year.

Fungal infection, psoriasis, and atopic dermatitis were the most common dermatological diseases among the seniors in the study (Table 3). Lesions were most frequently observed on the lower and upper extremities and the face (Table 4).

A majority of those questioned (63.9\%), including 55.9\% of PNH residents and $74.1 \%$ of U3A participants, visited a dermatologist, but only when it was necessary. More than a quarter of respondents (26.2\%) - 32.4\% of PNH residents and 18.5\% of U3A members - systematically visited a dermatologist's surgery. The same percentage of all respondents $(4.9 \%)$ did not seek a dermatologist's advice or applied self-therapy.

\section{Discussion}

Dry skin and itching are the most typical dermatological problems in the elderly, and constituted as much as $80.0 \%$ of all findings in this population group. ${ }^{4}$ Skin inflammations, such as eczema, psoriasis, and pruritic dermatosis, are relatively frequently reported. ${ }^{4,8}$ Though the diseases listed are not terminal, they are characterized by high morbidity and significantly decrease the life quality of the elderly, especially in the last months of their lives. ${ }^{8}$

The most frequent skin problem in the elderly in the world is eczema. ${ }^{9}$ Thapa et $\mathrm{al}^{9}$ carried out a study among 330 elderly patients in Nepal. The most frequent skin problem was also eczema (35.8\%). Similarly, eczema was the skin disease the most frequently diagnosed in the elderly in the
Table 3 Dermal diseases among respondents

\begin{tabular}{|c|c|c|c|c|}
\hline $\begin{array}{l}\text { Skin diseases diagnosed } \\
\text { in respondents }\end{array}$ & $\begin{array}{l}\text { PNH } \\
\text { residents }\end{array}$ & $\begin{array}{l}\text { U3A } \\
\text { students }\end{array}$ & Total & $P$-value \\
\hline Atopic dermatitis & & & & 0.124 \\
\hline \multicolumn{5}{|l|}{ Yes } \\
\hline $\mathrm{n}$ & 2 & 5 & 7 & \\
\hline$\%$ & $5.9 \%$ & $18.5 \%$ & $11.5 \%$ & \\
\hline \multicolumn{5}{|l|}{ No } \\
\hline $\mathrm{n}$ & 32 & 22 & 54 & \\
\hline$\%$ & $94.1 \%$ & $81.5 \%$ & $88.5 \%$ & \\
\hline \multicolumn{5}{|l|}{ Total } \\
\hline $\mathrm{n}$ & 34 & 27 & 61 & \\
\hline$\%$ & $100 \%$ & $100 \%$ & $100 \%$ & \\
\hline Vitiligo & & & & 0.696 \\
\hline \multicolumn{5}{|l|}{ Yes } \\
\hline $\mathrm{n}$ & 2 & I & 3 & \\
\hline$\%$ & $5.9 \%$ & $3.7 \%$ & $4.9 \%$ & \\
\hline \multicolumn{5}{|l|}{ No } \\
\hline $\mathrm{n}$ & 32 & 26 & 58 & \\
\hline$\%$ & $94.1 \%$ & $96.3 \%$ & $95.1 \%$ & \\
\hline \multicolumn{5}{|l|}{ Total } \\
\hline $\mathrm{n}$ & 34 & 27 & 61 & \\
\hline$\%$ & $100 \%$ & $100 \%$ & $100 \%$ & \\
\hline Furuncle & & & & 0.868 \\
\hline \multicolumn{5}{|l|}{ Yes } \\
\hline $\mathrm{n}$ & I & I & 2 & \\
\hline$\%$ & $2.9 \%$ & $3.7 \%$ & $3.3 \%$ & \\
\hline \multicolumn{5}{|l|}{ No } \\
\hline $\mathrm{n}$ & 33 & 26 & 59 & \\
\hline$\%$ & $97.1 \%$ & $96.3 \%$ & $96.7 \%$ & \\
\hline \multicolumn{5}{|l|}{ Total } \\
\hline $\mathrm{n}$ & 34 & 27 & 61 & \\
\hline$\%$ & $100 \%$ & $100 \%$ & $100 \%$ & \\
\hline Photodermatosis & & & & 0.369 \\
\hline \multicolumn{5}{|l|}{ Yes } \\
\hline $\mathrm{n}$ & 1 & 0 & 1 & \\
\hline$\%$ & $2.9 \%$ & 0 & $1.6 \%$ & \\
\hline \multicolumn{5}{|l|}{ No } \\
\hline $\mathrm{n}$ & 33 & 27 & 60 & \\
\hline$\%$ & $97.1 \%$ & $100 \%$ & $98.4 \%$ & \\
\hline \multicolumn{5}{|l|}{ Total } \\
\hline $\mathrm{n}$ & 34 & 27 & 61 & \\
\hline$\%$ & $100 \%$ & $100 \%$ & $100 \%$ & \\
\hline Fungal infection & & & & 0.396 \\
\hline \multicolumn{5}{|l|}{ Yes } \\
\hline $\mathrm{n}$ & 8 & 9 & 17 & \\
\hline$\%$ & $23.5 \%$ & $33.3 \%$ & $27.9 \%$ & \\
\hline \multicolumn{5}{|l|}{ No } \\
\hline $\mathrm{n}$ & 26 & 18 & 44 & \\
\hline$\%$ & $76.5 \%$ & $66.7 \%$ & $72.1 \%$ & \\
\hline \multicolumn{5}{|l|}{ Total } \\
\hline $\mathrm{n}$ & 34 & 27 & 61 & \\
\hline$\%$ & $100 \%$ & $100 \%$ & $100 \%$ & \\
\hline Epidermal cyst & & & & 0.107 \\
\hline \multicolumn{5}{|l|}{ Yes } \\
\hline $\mathrm{n}$ & 0 & 2 & 2 & \\
\hline$\%$ & 0 & $7.4 \%$ & $3.3 \%$ & \\
\hline No & & & & \\
\hline $\mathrm{n}$ & 34 & 25 & 59 & \\
\hline$\%$ & $100 \%$ & $92.6 \%$ & $96.7 \%$ & \\
\hline Total & & & & \\
\hline $\mathrm{n}$ & 34 & 27 & 61 & \\
\hline$\%$ & $100 \%$ & $100 \%$ & $100 \%$ & \\
\hline
\end{tabular}


Table 3 (Continued)

\begin{tabular}{|c|c|c|c|c|c|c|c|c|c|}
\hline $\begin{array}{l}\text { Skin diseases diagnosed } \\
\text { in respondents }\end{array}$ & $\begin{array}{l}\text { PNH } \\
\text { residents }\end{array}$ & $\begin{array}{l}\text { U3A } \\
\text { students }\end{array}$ & Total & $P$-value & $\begin{array}{l}\text { Skin diseases diagnosed } \\
\text { in respondents }\end{array}$ & $\begin{array}{l}\text { PNH } \\
\text { residents }\end{array}$ & $\begin{array}{l}\text { U3A } \\
\text { students }\end{array}$ & Total & $P$-value \\
\hline Lichen planus & & & & 0.868 & Shingles & & & & 0.937 \\
\hline Yes & & & & & Yes & & & & \\
\hline$n$ & 1 & 1 & 2 & & $\mathrm{n}$ & 4 & 3 & 7 & \\
\hline$\%$ & $2.9 \%$ & $3.7 \%$ & $3.3 \%$ & & $\%$ & $11.8 \%$ & $11.1 \%$ & $11.5 \%$ & \\
\hline No & & & & & No & & & & \\
\hline $\mathrm{n}$ & 33 & 26 & 59 & & $\mathrm{n}$ & 30 & 24 & 54 & \\
\hline$\%$ & $97.1 \%$ & $96.3 \%$ & $96.7 \%$ & & $\%$ & $88.2 \%$ & $88.9 \%$ & $88.5 \%$ & \\
\hline Total & & & & & Total & & & & \\
\hline$n$ & 34 & 27 & 61 & & $\mathrm{n}$ & 34 & 27 & 61 & \\
\hline$\%$ & $100 \%$ & $100 \%$ & $100 \%$ & & $\%$ & $100 \%$ & $100 \%$ & $100 \%$ & \\
\hline Dandruff & & & & 0.868 & Erysipelas & & & & 0.369 \\
\hline Yes & & & & & Yes & & & & \\
\hline $\begin{array}{l}n \\
\%\end{array}$ & $2.9 \%$ & $37 \%$ & $23 \%$ & & $\mathrm{n}$ & 0 & I & I & \\
\hline No & & & & & $\%$ & 0 & $3.7 \%$ & $1.6 \%$ & \\
\hline $\mathrm{n}$ & 33 & 26 & 59 & & No & & & & \\
\hline$\%$ & $97.1 \%$ & $96.3 \%$ & $96.7 \%$ & & $\mathrm{n}$ & 34 & 26 & 60 & \\
\hline Total & & & & & $\%$ & $100 \%$ & $96.3 \%$ & $98.4 \%$ & \\
\hline $\mathrm{n}$ & 34 & 27 & 61 & & Total & & & & \\
\hline$\%$ & $100 \%$ & $100 \%$ & $100 \%$ & & $\mathrm{n}$ & 34 & 27 & 61 & \\
\hline Psoriasis & & & & 0.560 & $\%$ & $100 \%$ & $100 \%$ & $100 \%$ & \\
\hline Yes & & & & & Erythema & & & & 0.868 \\
\hline $\mathrm{n}$ & 7 & 4 & II & & Yes & & & & \\
\hline$\%$ & $20.6 \%$ & $14.8 \%$ & $18 \%$ & & $\mathrm{n}$ & I & I & 2 & \\
\hline No & & & & & $\%$ & $2.9 \%$ & $3.7 \%$ & $3.3 \%$ & \\
\hline $\mathrm{n}$ & 27 & 23 & 50 & & No & & & & \\
\hline$\%$ & $79.4 \%$ & $85.2 \%$ & $82 \%$ & & $\mathrm{n}$ & 33 & 26 & 59 & \\
\hline Total & & & & & $\%$ & $97.1 \%$ & $96.3 \%$ & $96.7 \%$ & \\
\hline$n$ & 34 & 27 & 61 & & Total & & & & \\
\hline$\%$ & $100 \%$ & $100 \%$ & $100 \%$ & & $n$ & 34 & 27 & 61 & \\
\hline Balding & & & & 0.042 & $\begin{array}{l}\mathrm{n} \\
\%\end{array}$ & $100 \%$ & $100 \%$ & $100 \%$ & \\
\hline $\begin{array}{r}\text { Yes } \\
n\end{array}$ & 1 & 5 & 6 & & Ichthyosis & & & & 0.369 \\
\hline$\%$ & $2.9 \%$ & $18.5 \%$ & $9.8 \%$ & & Yes & & & & \\
\hline No & & & & & $\mathrm{n}$ & 1 & 0 & 1 & \\
\hline $\mathrm{n}$ & 33 & 22 & 55 & & $\%$ & $2.9 \%$ & 0 & $1.6 \%$ & \\
\hline$\%$ & $97.1 \%$ & $81.5 \%$ & $90.2 \%$ & & No & & & & \\
\hline Total & & & & & $\mathrm{n}$ & 33 & 27 & 60 & \\
\hline $\mathrm{n}$ & 34 & 27 & 61 & & $\%$ & $97.1 \%$ & $100 \%$ & $98.4 \%$ & \\
\hline$\%$ & $100 \%$ & $100 \%$ & $100 \%$ & & Total & & & & \\
\hline Angioma & & & & 0.254 & $\mathrm{n}$ & 34 & 27 & 61 & \\
\hline Yes & & & & & $\%$ & $100 \%$ & $100 \%$ & $100 \%$ & \\
\hline $\mathrm{n}$ & 4 & 1 & 5 & & Scabies & & & & 0.107 \\
\hline$\%$ & $11.8 \%$ & $3.7 \%$ & $8.2 \%$ & & Yes & & & & \\
\hline No & & & & & $\mathrm{n}$ & 2 & 0 & 2 & \\
\hline $\mathrm{n}$ & 30 & 26 & 56 & & $\%$ & $5.9 \%$ & 0 & $3.3 \%$ & \\
\hline$\%$ & $88.2 \%$ & $96.3 \%$ & $91.8 \%$ & & No & & & & \\
\hline Total & & & & & $n$ & 32 & 27 & 59 & \\
\hline$n$ & 34 & 27 & 61 & & $\%$ & $94.1 \%$ & $100 \%$ & $96.7 \%$ & \\
\hline$\%$ & $100 \%$ & $100 \%$ & $100 \%$ & & Total & & & & \\
\hline Rash & & & & 0.868 & $\mathrm{n}$ & 34 & 27 & 61 & \\
\hline $\begin{array}{r}\text { Yes } \\
n\end{array}$ & 1 & 1 & 2 & & $\%$ & $100 \%$ & $100 \%$ & $100 \%$ & \\
\hline$\%$ & $2.9 \%$ & $3.7 \%$ & $3.3 \%$ & & Acne & & & & 0.811 \\
\hline No & & & & & Yes & & & & \\
\hline$n$ & 33 & 26 & 59 & & $n$ & 2 & 2 & 4 & \\
\hline$\%$ & $97.1 \%$ & $96.3 \%$ & $96.7 \%$ & & $\%$ & $5.9 \%$ & $7.4 \%$ & $6.6 \%$ & \\
\hline Total & & & & & No & & & & \\
\hline$n$ & 34 & 27 & 61 & & $\mathrm{n}$ & 32 & 25 & 57 & \\
\hline$\%$ & $100 \%$ & $100 \%$ & $100 \%$ & & $\%$ & $94.1 \%$ & $92.6 \%$ & $93.4 \%$ & \\
\hline
\end{tabular}

Table 3 (Continued) 
Table 3 (Continued)

\begin{tabular}{|c|c|c|c|c|}
\hline $\begin{array}{l}\text { Skin diseases diagnosed } \\
\text { in respondents }\end{array}$ & $\begin{array}{l}\text { PNH } \\
\text { residents }\end{array}$ & $\begin{array}{l}\text { U3A } \\
\text { students }\end{array}$ & Total & $P$-value \\
\hline \multicolumn{5}{|l|}{ Total } \\
\hline $\mathrm{n}$ & 34 & 27 & 61 & \\
\hline$\%$ & $100 \%$ & $100 \%$ & $100 \%$ & \\
\hline Eczema & & & & 0.423 \\
\hline \multicolumn{5}{|l|}{ Yes } \\
\hline $\mathrm{n}$ & 1 & 2 & 3 & \\
\hline$\%$ & $2.9 \%$ & $7.4 \%$ & $4.9 \%$ & \\
\hline \multicolumn{5}{|l|}{ No } \\
\hline $\mathrm{n}$ & 33 & 25 & 58 & \\
\hline$\%$ & $97.1 \%$ & $92.6 \%$ & $95.1 \%$ & \\
\hline \multicolumn{5}{|l|}{ Total } \\
\hline $\mathrm{n}$ & 34 & 27 & 61 & \\
\hline$\%$ & $100 \%$ & $100 \%$ & $100 \%$ & \\
\hline Granuloma & & & & 0.369 \\
\hline \multicolumn{5}{|l|}{ Yes } \\
\hline $\mathrm{n}$ & 0 & 1 & I & \\
\hline$\%$ & 0 & $3.7 \%$ & $1.6 \%$ & \\
\hline \multicolumn{5}{|l|}{ No } \\
\hline $\mathrm{n}$ & 34 & 26 & 60 & \\
\hline$\%$ & $100 \%$ & $96.3 \%$ & $98.4 \%$ & \\
\hline \multicolumn{5}{|l|}{ Total } \\
\hline $\mathrm{n}$ & 34 & 27 & 61 & \\
\hline$\%$ & $100 \%$ & $100 \%$ & $100 \%$ & \\
\hline
\end{tabular}

Abbreviations: $\mathrm{PNH}$, public nursing home; U3A, University of the Third Age.

studies of Thaipisuttikul (34.9\%), ${ }^{4}$ Liao et al (34.9\%), ${ }^{5}$ and Yap et al (35.3\%)..$^{10}$ Among nearly 50,000 people of German origin, eczema was reported in $8.9 \%,{ }^{11}$ whereas in another earlier population study carried out in this country, it was reported in only $1.4 \% .^{12}$ In studies performed in Turkey, Tanzania, and Croatia, relatively low morbidity of eczema was reported $\left(11.1 \%, 11.9 \%\right.$, and $16.6 \%$, respectively). ${ }^{13-15}$ In Egypt, this skin problem constituted nearly $20.0 \%$ of all cases. ${ }^{16}$ In our study, eczema was found only in $4.9 \%$ of people. In another Polish study, ${ }^{17}$ eczema was reported in $15 \%$ of patients.

In our study, fungal infections constituted the main health problem $(27.9 \%)$. The incidence of these infections was confirmed by international examinations, ${ }^{9,10,14,18-20}$ showing that fungal infections were common among seniors. In Liao et al, ${ }^{5}$ fungal infections affected 6,429 elderly patients, which constituted $38 \%$ of the study population in Taiwan, in Abdel-Hafez et al ${ }^{16} 1,295$ people, ie, almost $1.2 \%$ of the study population, while in the study of Yap et $\mathrm{al}^{10}$ only $4.5 \%$ of Singapore inhabitants. A high index of morbidity of fungal infections (20.5\%) was established in the vicinity of Alexandria, in Egypt. ${ }^{21}$ In Iran, fungal infections affected 9.0\% of the study group, ${ }^{22}$ and in Tanzanian villages, morbidity was observed in the range of $4.0 \%-6.62 \% .^{23,24}$ In Ghana, the morbidity of fungal infections equaled $10.6 \%$, while in Great Britain it was only $1.9 \%{ }^{25}$ The cause of these differences
Table 4 Location of lesions among respondents

\begin{tabular}{|c|c|c|c|c|}
\hline $\begin{array}{l}\text { Where do you } \\
\text { have lesions? }\end{array}$ & $\begin{array}{l}\text { PNH } \\
\text { residents }\end{array}$ & $\begin{array}{l}\text { U3A } \\
\text { students }\end{array}$ & Total & $P$-value \\
\hline Head & & & & 0.585 \\
\hline \multicolumn{5}{|l|}{ Yes } \\
\hline $\mathrm{n}$ & II & 7 & 18 & \\
\hline$\%$ & $32.4 \%$ & $25.9 \%$ & $29.5 \%$ & \\
\hline \multicolumn{5}{|l|}{ No } \\
\hline $\mathrm{n}$ & 23 & 20 & 43 & \\
\hline$\%$ & $67.6 \%$ & $74.1 \%$ & $70.5 \%$ & \\
\hline \multicolumn{5}{|l|}{ Total } \\
\hline $\mathrm{n}$ & 34 & 27 & 61 & \\
\hline$\%$ & $100 \%$ & $100 \%$ & $100 \%$ & \\
\hline Face & & & & 0.873 \\
\hline \multicolumn{5}{|l|}{ Yes } \\
\hline $\mathrm{n}$ & 12 & 9 & 21 & \\
\hline$\%$ & $35.3 \%$ & $33.3 \%$ & $34.4 \%$ & \\
\hline \multicolumn{5}{|l|}{ No } \\
\hline $\mathrm{n}$ & 22 & 18 & 40 & \\
\hline$\%$ & $64.7 \%$ & $66.7 \%$ & $65 . \%$ & \\
\hline \multicolumn{5}{|l|}{ Total } \\
\hline $\mathrm{n}$ & 34 & 27 & 61 & \\
\hline$\%$ & $100 \%$ & $100 \%$ & $100 \%$ & \\
\hline Chest & & & & 0.937 \\
\hline \multicolumn{5}{|l|}{ Yes } \\
\hline $\mathrm{n}$ & 4 & 3 & 7 & \\
\hline$\%$ & $11.8 \%$ & $11.1 \%$ & $11.5 \%$ & \\
\hline \multicolumn{5}{|l|}{ No } \\
\hline $\mathrm{n}$ & 30 & 24 & 54 & \\
\hline$\%$ & $88.2 \%$ & $88.9 \%$ & $88.5 \%$ & \\
\hline \multicolumn{5}{|l|}{ Total } \\
\hline $\mathrm{n}$ & 34 & 27 & 61 & \\
\hline$\%$ & $100 \%$ & $100 \%$ & $100 \%$ & \\
\hline Back & & & & 0.321 \\
\hline \multicolumn{5}{|l|}{ Yes } \\
\hline $\mathrm{n}$ & 7 & 3 & 10 & \\
\hline$\%$ & $20.6 \%$ & $11.1 \%$ & $16.4 \%$ & \\
\hline \multicolumn{5}{|l|}{ No } \\
\hline $\mathrm{n}$ & 27 & 24 & 51 & \\
\hline$\%$ & $79.4 \%$ & $88.9 \%$ & $83.6 \%$ & \\
\hline \multicolumn{5}{|l|}{ Total } \\
\hline $\mathrm{n}$ & 34 & 27 & 61 & \\
\hline$\%$ & $100 \%$ & $100 \%$ & $100 \%$ & \\
\hline Hands & & & & 0.246 \\
\hline \multicolumn{5}{|l|}{ Yes } \\
\hline $\mathrm{n}$ & 15 & 8 & 23 & \\
\hline$\%$ & $44.1 \%$ & $29.6 \%$ & $37.7 \%$ & \\
\hline \multicolumn{5}{|l|}{ No } \\
\hline $\mathrm{n}$ & 19 & 19 & 38 & \\
\hline$\%$ & $55.9 \%$ & $70.4 \%$ & $62.3 \%$ & \\
\hline \multicolumn{5}{|l|}{ Total } \\
\hline $\mathrm{n}$ & 34 & 27 & 61 & \\
\hline$\%$ & $100 \%$ & $100 \%$ & $100 \%$ & \\
\hline Legs & & & & $0.47 \mid$ \\
\hline \multicolumn{5}{|l|}{ Yes } \\
\hline $\mathrm{n}$ & 17 & 11 & 28 & \\
\hline$\%$ & $50 \%$ & $40.7 \%$ & $45.9 \%$ & \\
\hline No & & & & \\
\hline $\mathrm{n}$ & 17 & 16 & 33 & \\
\hline$\%$ & $50 \%$ & $59.3 \%$ & $54.1 \%$ & \\
\hline Total & & & & \\
\hline $\mathrm{n}$ & 34 & 27 & 61 & \\
\hline$\%$ & $100 \%$ & $100 \%$ & $100 \%$ & \\
\hline
\end{tabular}


Table 4 (Continued)

\begin{tabular}{|c|c|c|c|c|}
\hline $\begin{array}{l}\text { Where do you } \\
\text { have lesions? }\end{array}$ & $\begin{array}{l}\text { PNH } \\
\text { residents }\end{array}$ & $\begin{array}{l}\text { U3A } \\
\text { students }\end{array}$ & Total & $P$-value \\
\hline Sexual organs & & & & 0.696 \\
\hline \multicolumn{5}{|l|}{ Yes } \\
\hline $\mathrm{n}$ & 2 & 1 & 3 & \\
\hline$\%$ & $5.9 \%$ & $3.7 \%$ & $4.9 \%$ & \\
\hline \multicolumn{5}{|l|}{ No } \\
\hline$n$ & 32 & 26 & 58 & \\
\hline$\%$ & $94.1 \%$ & $96.3 \%$ & $95.1 \%$ & \\
\hline \multicolumn{5}{|l|}{ Total } \\
\hline $\mathrm{n}$ & 34 & 27 & 61 & \\
\hline$\%$ & $100 \%$ & $100 \%$ & $100 \%$ & \\
\hline Groin & & & & 0.422 \\
\hline \multicolumn{5}{|l|}{ Yes } \\
\hline $\mathrm{n}$ & 3 & 1 & 4 & \\
\hline$\%$ & $8.8 \%$ & $3.7 \%$ & $6.6 \%$ & \\
\hline \multicolumn{5}{|l|}{ No } \\
\hline $\mathrm{n}$ & 31 & 26 & 57 & \\
\hline$\%$ & $91.2 \%$ & $96.3 \%$ & $93.4 \%$ & \\
\hline \multicolumn{5}{|l|}{ Total } \\
\hline$n$ & 34 & 27 & $6 I$ & \\
\hline$\%$ & $100 \%$ & $100 \%$ & $100 \%$ & \\
\hline Nails & & & & 0.274 \\
\hline \multicolumn{5}{|l|}{ Yes } \\
\hline$n$ & 5 & 7 & 12 & \\
\hline$\%$ & $14.7 \%$ & $25.9 \%$ & $19.7 \%$ & \\
\hline \multicolumn{5}{|l|}{ No } \\
\hline$n$ & 29 & 20 & 49 & \\
\hline$\%$ & $85.3 \%$ & $74.1 \%$ & $80.3 \%$ & \\
\hline \multicolumn{5}{|l|}{ Total } \\
\hline $\mathrm{n}$ & 34 & 27 & 61 & \\
\hline$\%$ & $100 \%$ & $100 \%$ & $100 \%$ & \\
\hline
\end{tabular}

Abbreviations: PNH, public nursing home; U3A, University of the Third Age.

between different parts of the world could be specific climatic and prophylactic factors of these communities.

In our study, the second disease with regard to incidence was psoriasis. In Reszke et al, ${ }^{26}$ in patients of the Department of Dermatology and Geriatrics of the Medical University of Wrocław, 69 patients (38.5\%) were diagnosed with this disease. Other research ${ }^{17}$ performed in Poland confirmed the higher morbidity of psoriasis among Polish seniors (10.8\%) compared to exotic countries, such as Singapore and Taiwan. In the Singapore study, ${ }^{10}$ this problem affected fewer patients and constituted $3.1 \%$ of all confirmed skin diseases. A similar percentage of cases was reported in Taiwan (3.9\%). ${ }^{5}$ In the German study, the percentage of people with psoriasis was lower and equaled 2\%, ${ }^{11}$ and in study carried in Egypt only $0.19 \%$ of all diagnosed skin diseases, which equaled 15 clinical cases. ${ }^{16}$ The morbidity of psoriasis is markedly higher in the USA and European countries, ${ }^{27}$ and it has been proved that Caucasians are more prone to develop this skin problem. ${ }^{28}$ The standard example illustrating this correlation are the studies of Doe et al, ${ }^{25}$ carried out in hospitals in the UK and Ghana: in the British hospital, $6.2 \%$ of psoriasis cases were reported, whereas in Ghana only $0.4 \% .{ }^{25}$ Summing up, in the case of psoriasis, genetic and environmental factors are the main determinant.

\section{Conclusion}

Skin diseases pose a significant health problem in the senior population of Bialystok. Fungal infection is the most frequently reported skin disease, so seniors should be educated how to have a hygienic lifestyle. There is a need to encourage the elderly to seek dermatological advice offered by medical specialists.

\section{Disclosure}

The authors report no conflicts of interest in this work.

\section{References}

1. Grove GL. Physiologic changes in older skin. Clin Geriatr Med. 1989;5: 115-125.

2. Carter DM, Balin AK. Dermatological aspects of aging skin. Med Clin North Am. 1983;67:531-543.

3. Mathus-Vliegen EM. Old age, malnutrition, and pressure sores: an ill-fated alliance. J Gerontol A Biol Sci Med Sci. 2004;59:355-360.

4. Thaipisuttikul Y. Pruritic skin diseases in the elderly. J Dermatol. 1998;25:153-157.

5. Liao YH, Chen KH, Tseng MP, Sun CC. Pattern of skin diseases in geriatric patients in Taiwan: a 7-year survey from outpatient clinic to a university medical center. Dermatology. 2001;203:308-313.

6. Kleinsmith DM, Perricone NV. Common skin problems in the elderly. Dermatol Clin. 1986;4:485-499.

7. Loo WJ, Burrows NP. Management of autoimmune skin disorders in the elderly. Drugs Aging. 2004;21:767-777.

8. Gilchrest BA, Chiu N. Common skin disorders. In: Beers MH, Berkow R, editors. The Merck Manual of Geriatrics. 3rd ed. Whitehouse Station, NJ: Merck and Co Inc; 2000:1238-1260.

9. Thapa DP, Jha AK, Kharel C, Shrestha S. Dermatological problems in geriatric patients: a hospital based study. Nepal Med Coll J. 2012; 14:193-195.

10. Yap KB, Siew MG, Goh CL. Pattern of skin diseases in the elderly seen at the National Skin Centre (Singapore) 1990. Singapore Med J. 1994; 35:147-150.

11. Schaefer I, Rustenbach SJ, Zimmer L, Augustin M. Prevalence of skin diseases in a cohort of 48,665 employees in Germany. Dermatology. 2008;217:169-172.

12. Wolkewitz M, Rothenbacher D, Löw M, et al. Lifetime prevalence of self-reported atopic diseases in a population-based sample of elderly subjects: results of the ESTHER study. Br J Dermatol. 2007;156: 693-697.

13. Fleischer AB. Pruritus in the elderly. Adv Dermatol. 1995;10:41-60.

14. Kiliç A, Gül U, Aslan E, Soylu S. Dermatological findings in the senior population of nursing homes in Turkey. Arch Gerontol Geriatr. 2008;47: 93-98.

15. Cvitanović H, Knezević E, Kuljanac I, Jancić E. Skin disease in a geriatric patients group in outpatient dermatologic clinic: Karlovac, Croatia. Coll Antropol. 2010;34 Suppl 2:247-251.

16. Abdel-Hafez K, Abdel-Aty MA, Hofny ER. Prevalence of skin diseases in rural areas of Assiut Governorate, Upper Egypt. Int J Dermatol. 2003; 42:887-892.

17. Pawlaczyk M, Zukowska E. [Analysis of dermatoses treated at the dermatological ward in elderly patients]. Geriatria. 2011;5:94-98. Polish. 
18. Souissi A, Zeglaoui F, El Fekih N, Fazaa B, Zouari B, Kamoun MR. [Skin diseases in the elderly: a multicentre Tunisian study]. Ann Dermatol Venereol. 2006;133:231-234. French.

19. Furue M, Yamazaki S, Jimbow K, et al. Prevalence of dermatological disorders in Japan: a nationwide, cross-sectional, seasonal, multicenter, hospital-based study. J Dermatol. 2011;38:310-320.

20. Yalçin B, Tamer E, Toy GG, Oztaş P, Hayran M, Alli N. The prevalence of skin diseases in the elderly: analysis of 4099 geriatric patients. Int $J$ Dermatol. 2006;45:672-676.

21. Khairy A. The Role of Some Ecological Factors in Determining the Skin Lesions in a Rural Area in the ARE [doctoral thesis]. Alexandria: Alexandria University; 2001.

22. Omidynia E, Farshchian M, Sadjjadi M, Zamanian A, Rashidpouraei R. A study of dermatophytoses in Hamadan, the government-ship of West Iran. Mycopathologia. 1996;133:9-13.
23. Gibbs S. Skin disease and socioeconomic conditions in rural Africa: Tanzania. Int J Dermatol. 1996;35:633-639.

24. Henderson C. Skin disease in rural Tanzania. Int J Dermatol. 1996;35: 640-642.

25. Doe PT, Asiedu A, Acheampong JW, Payne CM. Skin diseases in Ghana and the UK. Int J Dermatol. 2001;40:323-326.

26. Reszke R, Pełka D, Walasek A, Machaj Z, Reich A. Skin disorders in elderly subjects. Int J Dermatol. 2015;54:e332-e338.

27. Nevitt G, Hutchinson P. Psoriasis in the community: prevalence, severity and patients' beliefs and attitudes towards the disease. Br J Dermatol. 1996;135:533-537.

28. Farber E, Nall L. Psoriasis in the tropics: epidemiologic, genetic, clinical and therapeutic aspects. Dermatol Clin. 1994;12:805-816.
Clinical Interventions in Aging

\section{Publish your work in this journal}

Clinical Interventions in Aging is an international, peer-reviewed journal focusing on evidence-based reports on the value or lack thereof of treatments intended to prevent or delay the onset of maladaptive correlates of aging in human beings. This journal is indexed on PubMed Central, MedLine,

\section{Dovepress}

CAS, Scopus and the Elsevier Bibliographic databases. The manuscript management system is completely online and includes a very quick and fair peer-review system, which is all easy to use. Visit http://www.dovepress. $\mathrm{com} /$ testimonials.php to read real quotes from published authors. 